\title{
Whole outline scanning measurement of internal gear by using CNC gear measuring machine
}

\author{
Syuhei Kurokawa ${ }^{1, *}$, Yuuki Utsunomiya ${ }^{1}$, Terutake Hayashi ${ }^{1}$, Tetsuya Taguchi $^{2}$, and Ryota Matsuoka ${ }^{2}$ \\ ${ }^{1}$ Kyushu University, Department of Mechanical Engineering, 819-0395 Fukuoka, Japan \\ ${ }^{2}$ Osaka Seimitsu Kikai Co., Ltd., Manufacturing Division, 577-0032 Higashi-Osaka, Japan
}

\begin{abstract}
A three-dimensional probe is mounted on a specialized gear measuring machine whose measurement target is limited on the working flanks. The newly developed measuring machine has a coordinate measuring mechanism, aiming to be a highly versatile and relatively inexpensive measuring machine. According to our previous research, in addition to the working tooth surface, shape data of the root, bottom and tip profiles can be acquired with a single measurement operation. However, the target object for which this developed method has been applied was only the external cylindrical gear. In this paper, we applied and expanded the development method to the internal cylindrical gear and the evaluation of the axial displacement in the same cross-sectional scanning of the internal helical gear are performed.
\end{abstract}

\section{Introduction}

In manufacturing gears, machining and inspection are inextricably linked together, and to improve the accuracy of gears, it is essential to improve not only machining technology but also high-precision inspection technology [1][2]. The purpose of this research is to develop a versatile and relatively inexpensive gear measuring machine. According to our previous research, a gear outline scanning measurement was developed, in which a $3 \mathrm{D}$ probe is mounted on a $\mathrm{CNC}$ gear measurement machine and the probe performs scanning measurement with a single path without detaching the stylus from the measured surface [3][4]. By this method, it is possible to add the measurement function of the tooth root, the tooth bottom and the tooth tip profiles to the measuring machine whose measurement range is conventionally limited to the working tooth flank. However, the target gear to which this measurement method was applied was only the external cylindrical gear. It is necessary to adapt this method to different types of gears to improve the versatility of the developed machine.

In this paper, this development method is applied to the internal cylindrical gear, and acquisition of the whole outline data and evaluation of the axial displacement in the same cross-sectional scanning of the internal helical gear are performed.

\section{Overview of developed measuring machine}

We used the $\mathrm{CNC}$ full-automatic gear measurement machine (CLP-35DDS, Osaka Seimitsu Kikai Co., Ltd., Japan) as a gear measurement machine to be developed. This measuring machine is a base circle adjustment type
$\mathrm{CNC}$ gear measuring machine for involute gears, and it is possible to measure the tooth profile, lead and pitch deviations, which correspond to the accuracy of the working tooth flank of involute cylindrical gears.

\subsection{Drive units}

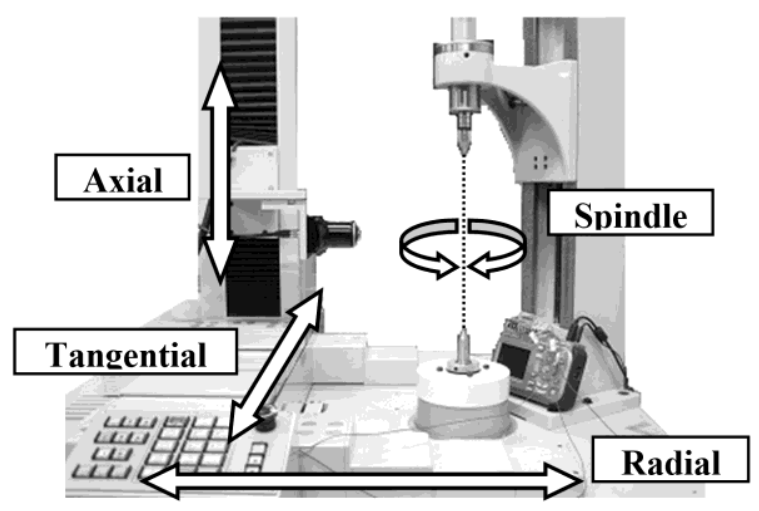

Fig. 1. Developed gear measuring machine.

The developed measuring machine has a rotating spindle that mounts the gear to be measured and a three-axis orthogonal slide that positions the stylus. Figure 1 shows the appearance of the measuring machine and a schematic diagram of each axis. The main axis of rotation is called the spindle axis, and the orthogonal slide consists of the radial direction slide, the tangential direction slide, and the axial direction slide in the gear rotational axis direction. Each axis can be translated independently of each other. The motor commands can set the speed and movement distance by giving the driver pulse values and their frequency. A torque motor

Corresponding author: kurobe-@mech.kyushu-u.ac.jp 
is used for the spindle axis, a servomotor and a ball screw for the radial axis as well as the axial axis, and a linear motor for the tangential axis. A rotary encoder is used to detect the position of the spindle axis, and a linear encoder is used to detect the position of the radial, tangential, and axial axes, respectively. The resolution is $0.18^{\prime \prime}$ for the spindle axis, $0.1 \mu \mathrm{m}$ for the radial axis, 0.05 $\mu \mathrm{m}$ for the tangential axis, and $0.1 \mu \mathrm{m}$ for the axial axis. Also, the radial axis and the axial axis are controlled by semi-closed loops, the tangential axis and the spindle axis are controlled by full-closed loops.

\subsection{Probing system}

A 3D probe (SP600Q, RENISHAW) is used to measure the whole outline including the tooth root, tooth bottom and tooth tip of a gear. This probe is an analog tactile scanning probe and can detect displacement in three axial directions simultaneously. When the stylus tip comes in contact with the measurement surface, it is displaced in the direction in which the reaction force is received, and the displacement is converted to a voltage. In addition, this probe has a structure in which the detector part and the stylus part can be separated, so the stylus can be replaced according to the measurement object. The measurement rage of the probe is $\pm 1.0 \mathrm{~mm}$, the resolution is $0.1 \mu \mathrm{m}$, and the spring rate is $1.2 \mathrm{~N} / \mathrm{mm}$ in every direction. The appearance of the probe is shown in Fig. 2.

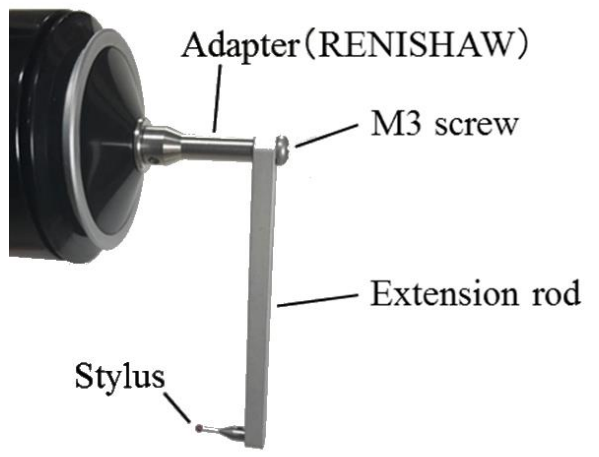

Fig. 2. Probing system.

In order to measure internal helical gears and multi cross-sectional measurement, a stylus must be mounted in the radial direction of the gears. Therefore, the extension rod combined with the adapter is used as shown in Fig.2. The stylus has a nominal tip diameter of $1.5 \mathrm{~mm}$ made from an artificial ruby.

\section{Whole outline scanning measurement}

In the conventional gear specialized measuring machine, the measuring region is limited to the working tooth flank, and it is difficult to measure the shape at the tooth root, tooth bottom and tooth tip. In addition, the measurement is conducted individually for each gear tooth with the index motion, and the measurement results are also output individually, and it takes time to measure all the teeth. In order to solve these problems, the development machine uses the three-dimensional scanning probe mentioned in the previous section to synchronize the movement of the probe in the radial direction and around the spindle axis so that the stylus tip does not detach from the gear surface to be measured [2]. The schematic diagram of the measurement is sown in Fig.3. This method is referred to as the whole outline gear scanning measurement in this paper.

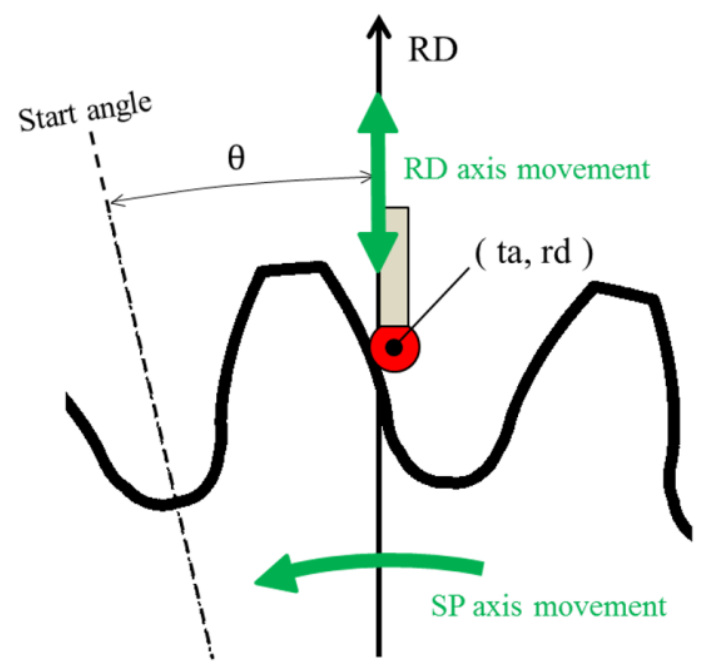

Fig. 3. Synchronous motion of the radial (RD) and the spindle (SP) movement.

The advantage of the whole outline scanning measurement is that it becomes possible to acquire the shape data of all the teeth including the tooth root, the tooth bottom and the tooth tip by a single scanning measurement. Furthermore, the measurement time can be shortened significantly without index motion.

The scanning method adopts the predefined path scanning using the target points for the object surface to be measured. The target points are calculated by the point-to-point intermittent touching motion in the radial direction. It is referred to as a rough measurement, which can be conducted even without gear specifications. The rough measurement is performed for one tooth space, and the rotational coordinate transformation is used to create all the target points for the whole outline of the target gear.

\section{Test gears}

There are two types of internal test gears: a spur gear and a helical gear, both with a module of $4 \mathrm{~mm}$, the normal pressure angle of $20^{\circ}$, the number of teeth of 45 and the facewith of $40 \mathrm{~mm}$. The helix angle of the internal helical gear is $20^{\circ}$. An appearance of the enlarged view of the internal test gears is shown in Fig. 4. and the attachment to the measuring machine is shown in Fig.5. As can be seen from the photograph, each test gear is integrated with a fixture shaft to reduce the amount of eccentricity when attached to the center of the measuring machine. 
The test gears were manufactured with high accuracy, and the accuracy inspection was performed using the highest precision measuring machine at the gear inspection site of Osaka Seimitsu Kikai Co., Ltd. The accuracy inspection was performed by measuring and evaluating the deviations of the working tooth flank. By means of the measurement accuracy of the measuring machine used for the accuracy inspection, the shape deviations of the test gears are guaranteed to be within $\pm 1 \mu \mathrm{m}$ from the evaluated results. Therefore, the test gears can be used to verify the accuracy of the whole outline scanning measurement.

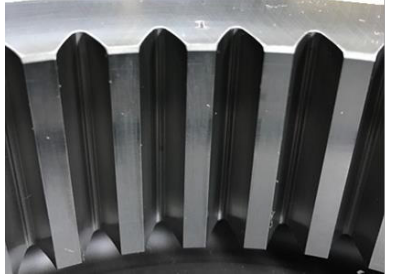

(a) Spur gear

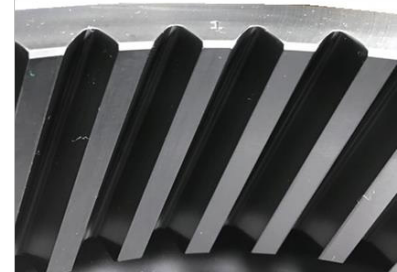

(b) Helical gear
Fig. 4. Enlarged view of the internal test gears.

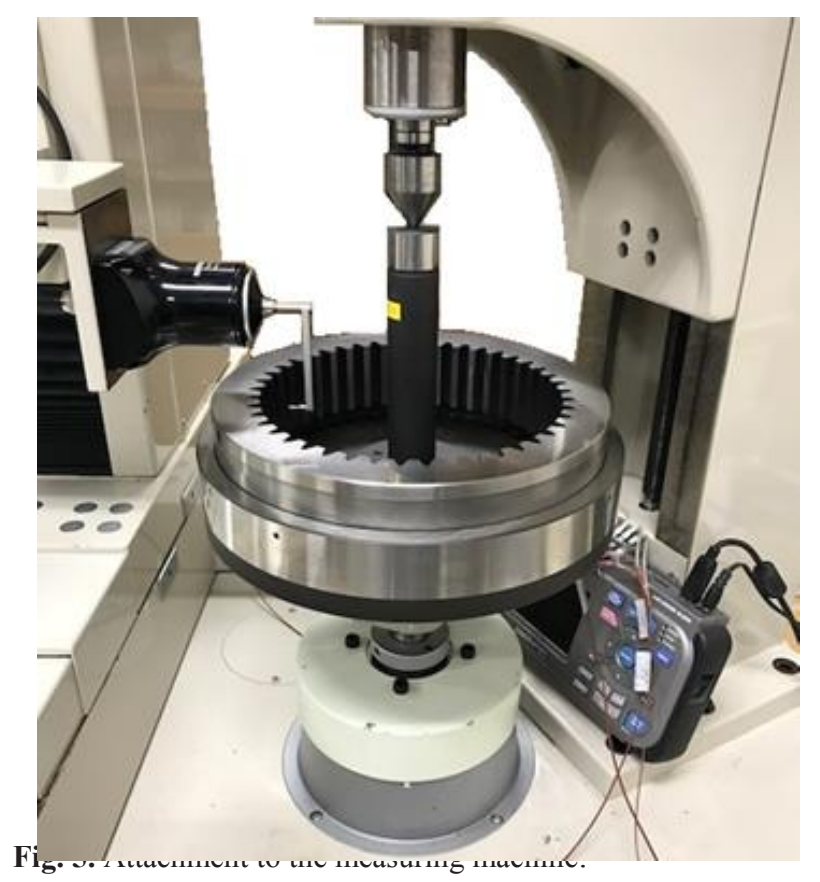

\section{Measurement results and discussions}

\subsection{Results in internal spur gear}

Figure 6 shows the measurement results of the internal spur gear. By adapting the whole outline scanning measurement to the internal gear, profile data of the tooth root, tooth bottom and tooth tip of all teeth were acquired at one time scanning in addition to the working tooth flanks. The scanning speed was $1 \mathrm{~mm} / \mathrm{s}$, and the actual measurement time was about 25 minutes. Since the measurement time of only the tooth profile deviations by the conventional method is about 50 minutes at the same scanning speed of $1 \mathrm{~mm} / \mathrm{s}$, the measurement time can be significantly shortened.

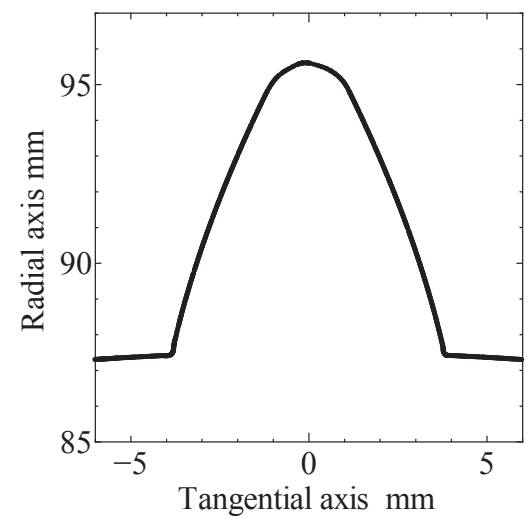

(a) The 1 st tooth

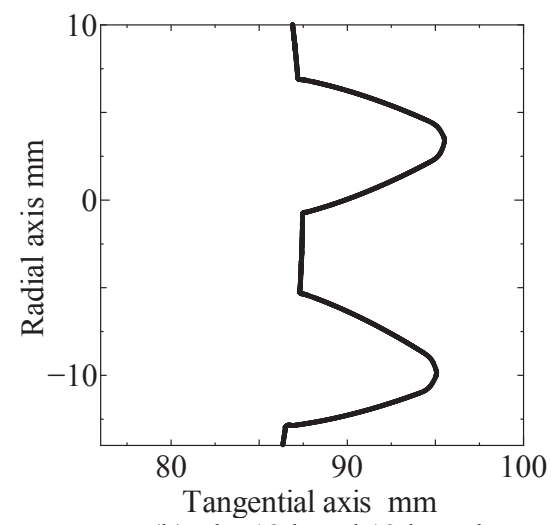

(b) The 12th and 13th teeth

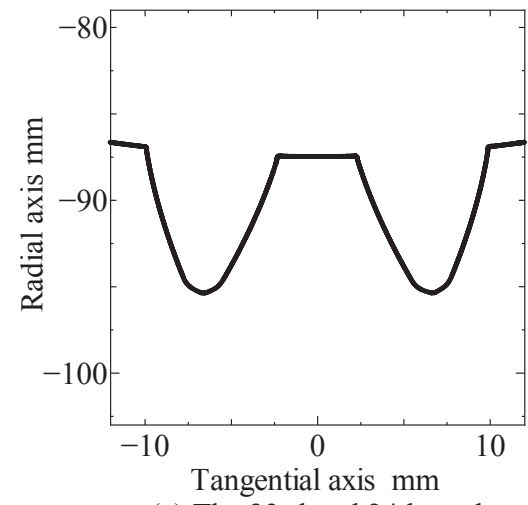

(c) The 23rd and 24th teeth

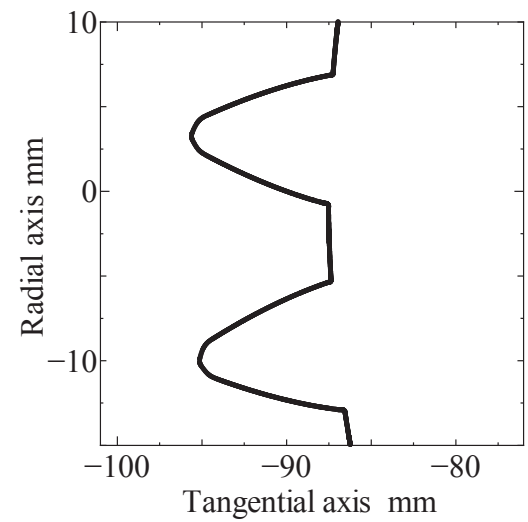

(d) The 34th and 35th teeth

Fig. 6. Measured results for the internal spur gear. 


\subsection{Measurement of internal helical gear}

In the spur gear cross-sectional scanning measurement, the motion was based on the two-axis operation of the radial and the spindle axes, which results in the stylus movement in the two-dimensional horizontal direction along the tangential and the radial axes. When the helical gear is measured, the stylus moves also along the axial direction due to the helix angle of the tooth surface. If the stylus touches on the helical gear surface in the horizontal direction, the stylus slips along the tooth surface and deviates from the target axial cross-section.

It is possible to measure the intended cross-sections of helical gears by setting the three-dimensional target points in consideration of the helix angle and performing the scanning measurement by the three-axis operation as shown in Fig.7. To obtain all the target points for the scanning measurement, the helix angles in arbitrary position on the tooth surface must be known even at the tooth root and bottom regions. The method has been used to acquire the helix angles by measuring one tooth space with the two-axis operation by obtaining the amount of axial deviations $d_{\mathrm{ax}}$ expressed by Eq. (1).

$$
d_{\mathrm{ax}}=d_{3 \text { Dprobe }} \cdot \sin \beta
$$

where $d_{3 \text { Dprobe }}$ is given measurement displacement and $\beta$ is an arbitrary helix angle to be identified. Since the measurement of two-axis operation is conducted only for one tooth space just in preparation of the target points, the total measurement time does not increase significantly.

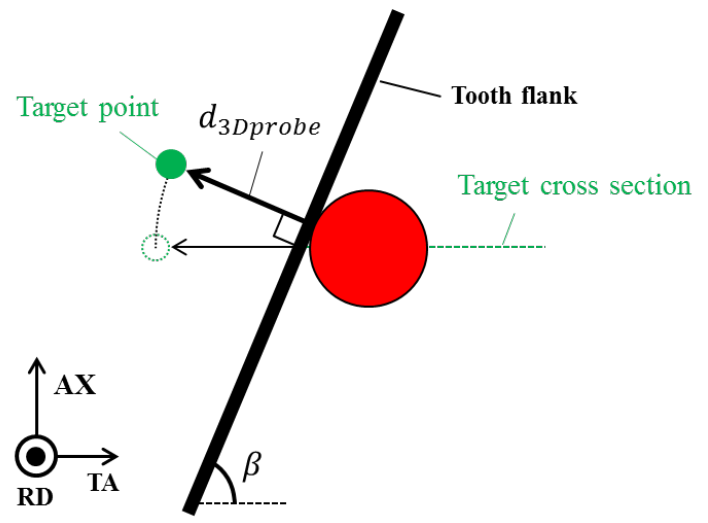

Fig. 7. Approaching direction of the stylus in helical gear measurement.

\subsection{Results in internal helical gear}

The test internal helical gear was measured at a scanning speed of $1.0 \mathrm{~mm} / \mathrm{s}$, a given measurement displacement of $300 \mu \mathrm{m}$, and the number of target points of 50 points per tooth space. The measurement was made by both twoaxis and three-axis operations for comparison of reduction of axial displacement of the stylus during scanning.

Figure 8 shows an example of the measurement results for the 1st tooth as representative, and Fig.9 shows the axial deviations from the target cross-section.

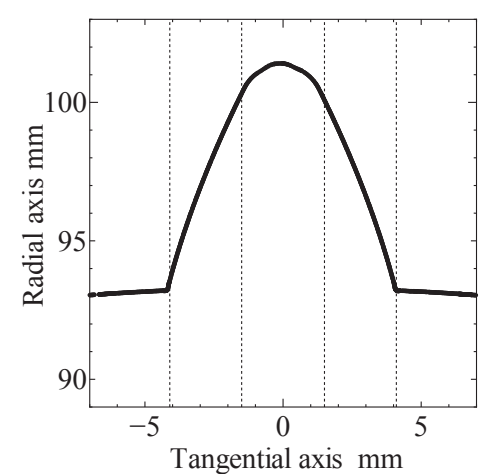

Fig. 8. Measured result for the internal helical gear for the 1st tooth.

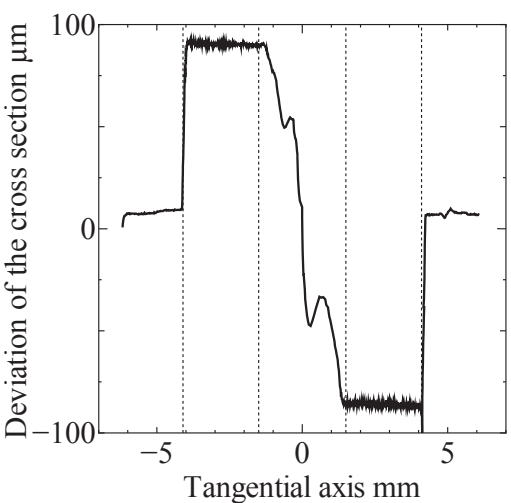

(a) The two-axis motion

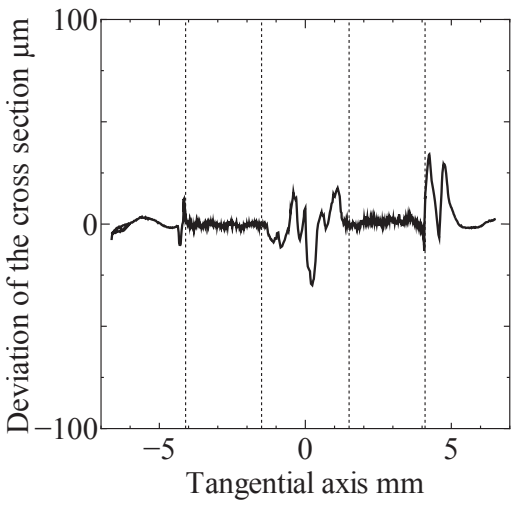

(b) The three-axis motion

Fig. 9. Axial deviations from the target cross-section.

At the time of measurement in the two-axis operation, the maximum axial deviation from the target cross-section is about $100 \mu \mathrm{m}$ on both right and left working flanks. On the other hand, in the measurement by three-axis operation, it is possible to reduce the amount of axial deviations on both working flanks to 10 $\mu \mathrm{m}$ or less. This value is the same as in the external gear measurement, and it can be confirmed that the same cross-sectional scanning measurement of the helical gear by the three-axis operation is possible even in the internal gear measurement using the stylus extension.

\section{Conclusions}

The gear measurement was applied to the internal gear, making scanning measurements with a single path 
without detaching the stylus from the tooth surface. The whole outline scanning of the internal spur gear and the internal helical gear was conducted, and the shape data of all the teeth including the tooth root, the tooth bottom and the tooth tip profiles could be acquired. In addition, it has been confirmed that the same cross-sectional scanning measurement of the helical gear by three-axis operation is also applicable to the internal gear measurement using a stylus extension.

\section{References}

1. J. TANG, Precision Engineering, 45 (2016)

2. G. KOULIN, Meas. Sci. Technol., 28 (2017)

3. S. KUROKAWA, H. KIDO, T. TAGUCHI, T. OKADA, O. OHNISHI, and T. DOI, Applied Mechanics and Materials, 86, 838-841 (2011)

4. S. KUROKAWA, T. TERAOKA, Y. UTSUNOMIYA, T. TAGUCHI, T. HAYASHI, and Y. MATSUKAWA, Proc. of the JSME Int. Conf. on Motion and Power Transmissions, Kyoto, 179-182 (2017) 